\title{
GAUSSIAN HYPERGEOMETRIC SERIES AND SUPERCONGRUENCES
}

\author{
ROBERT OSBURN AND CARSTEN SCHNEIDER
}

\begin{abstract}
Let $p$ be an odd prime. In 1984, Greene introduced the notion of hypergeometric functions over finite fields. Special values of these functions have been of interest as they are related to the number of $\mathbb{F}_{p}$ points on algebraic varieties and to Fourier coefficients of modular forms. In this paper, we explicitly determine these functions modulo higher powers of $p$ and discuss an application to supercongruences. This application uses two non-trivial generalized Harmonic sum identities discovered using the computer summation package Sigma. We illustrate the usage of Sigma in the discovery and proof of these two identities.
\end{abstract}

\section{INTRODUCTION}

In [16 and 17, Greene defined general hypergeometric series over finite fields. His aim was to show that these functions satisfy properties analogous to classical hypergeometric series. For example, the four major evaluations of the ordinary hypergeometric series ${ }_{3} F_{2}$ due to Saalschütz, Dixon, Watson, and Whipple 5 all have finite field interpretations (see page 126 of [16]). Greene's work was in part motivated by the analogy between Gauss sums and Gamma functions [10, 23], 43.

His approach has proven to be a powerful technique for character sum evaluations. Recently, several authors have shown that special values of these functions are related to the number of points over $\mathbb{F}_{p}, p$ an odd prime, of Calabi-Yau threefolds [3], traces of Hecke operators [11, formulas for Ramanujan's $\tau$-function [35, and the number of points on a family of elliptic curves [13]. We should also mention that hypergeometric series over arbitrary fields has been developed [14, [15], but their application to number theory has yet to be investigated.

The purpose of this paper is to further study arithmetic properties of hypergeometric functions over finite fields. In particular, we explicitly determine these functions modulo higher powers of $p$ and then briefly discuss extensions of supercongruences.

We first recall some definitions. Let $\mathbb{F}_{p}$ denote the finite field with $p$ elements. We extend all characters $\chi$ of $\mathbb{F}_{p}^{*}$ to $\mathbb{F}_{p}$ by setting $\chi(0):=0$. Following [16] and [17, we give two definitions. The first definition is the finite field analogue of the

Received by the editor April 23, 2007 and, in revised form, November 1, 2007.

2000 Mathematics Subject Classification. Primary 11F33, 33F10; Secondary 11S80.

The second author was supported by the SFB-grant F1305 and the grant P16613-N12 of the Austrian FWF. 
binomial coefficient. If $A$ and $B$ are characters of $\mathbb{F}_{p}$, then

$$
\left(\begin{array}{l}
A \\
B
\end{array}\right):=\frac{B(-1)}{p} J(A, \bar{B})=\frac{B(-1)}{p} \sum_{x \in \mathbb{F}_{p}} A(x) \bar{B}(1-x),
$$

where $J(\chi, \psi)$ denotes the Jacobi sum if $\chi$ and $\psi$ are characters of $\mathbb{F}_{p}$. The second definition is the finite field analogue of ordinary hypergeometric functions. If $A_{0}$, $A_{1}, \ldots, A_{n}$, and $B_{1}, \ldots, B_{n}$ are characters of $\mathbb{F}_{p}$, then the Gaussian hypergeometric function over $\mathbb{F}_{p}$ is defined by

$$
{ }_{n+1} F_{n}\left(\begin{array}{cccc}
A_{0}, & A_{1}, & \ldots, & A_{n} \\
& B_{1}, & \ldots, & B_{n}
\end{array} \mid x\right)_{p}:=\frac{p}{p-1} \sum_{\chi}\left(\begin{array}{c}
A_{0} \chi \\
\chi
\end{array}\right)\left(\begin{array}{c}
A_{1} \chi \\
B_{1} \chi
\end{array}\right) \ldots\left(\begin{array}{c}
A_{n} \chi \\
B_{n} \chi
\end{array}\right) \chi(x),
$$

where the summation is over all characters $\chi$ of $\mathbb{F}_{p}$. In this paper, we restrict our attention to the case $A_{i}=\phi_{p}$ for all $i$ and $B_{j}=\epsilon_{p}$ for all $j$ where $\phi_{p}$ is the quadratic character and $\epsilon_{p}$ is the trivial character $\bmod p$. We shall denote this value by ${ }_{n+1} F_{n}(\lambda)$. By [16] and [17, $p^{n}{ }_{n+1} F_{n}(\lambda) \in \mathbb{Z}$. Before stating the main result, we recall that for $i, n \in \mathbb{N}$, generalized Harmonic sums $H_{n}^{(i)}$ are defined by

$$
H_{n}^{(i)}:=\sum_{j=1}^{n} \frac{1}{j^{i}}
$$

and $H_{0}^{(i)}:=0$. We now define the quantities

$$
\begin{aligned}
X(p, \lambda, n) & :=\phi_{p}(\lambda) \sum_{j=0}^{\frac{p-1}{2}}\left(\begin{array}{c}
\frac{p-1}{2}+j \\
j
\end{array}\right)^{l}\left(\begin{array}{c}
\frac{p-1}{2} \\
j
\end{array}\right)^{l}(-1)^{j l} \lambda^{-j}\left(1+2(n+1) j\left(H_{\frac{p-1}{2}+j}^{(1)}-H_{j}^{(1)}\right)\right. \\
& \left.+j^{2}\left(\frac{n+1}{2}(1+n)\left(H_{\frac{p-1}{2}+j}^{(1)}-H_{j}^{(1)}\right)^{2}-\left(\frac{n+1}{2}\right)\left(H_{\frac{p-1}{2}+j}^{(2)}-H_{j}^{(2)}\right)\right)\right),
\end{aligned}
$$

$$
\begin{gathered}
Y(p, \lambda, n):=\phi_{p}(\lambda) \sum_{j=0}^{\frac{p-1}{2}}\left(\begin{array}{c}
\frac{p-1}{2}+j \\
j
\end{array}\right)^{l}\left(\begin{array}{c}
\frac{p-1}{2} \\
j
\end{array}\right)^{l}(-1)^{j l} \lambda^{-j p} \\
\cdot\left(1+(n+1) j\left(H_{\frac{p-1}{2}+j}^{(1)}-H_{j}^{(1)}\right)-\left(\frac{n+1}{2}\right) j\left(H_{\frac{p-1}{2}+j}^{(1)}-H_{\frac{p-1}{2}-j}^{(1)}\right)\right), \\
Z(p, \lambda, n):=\phi_{p}(\lambda) \sum_{j=0}^{\frac{p-1}{2}}\left(\begin{array}{c}
2 j \\
j
\end{array}\right)^{2 l} 16^{-j l} \lambda^{-j p^{2}},
\end{gathered}
$$

and

$$
D(p, \lambda):=\sum_{j=0}^{\frac{p-5}{2}} \frac{j !^{2}}{\prod_{i=0}^{j}\left(i+\frac{1}{2}\right)^{2}}(j+1)^{2} \lambda^{-j-1},
$$

where $l:=\frac{n+1}{2}$. The main result of this paper is the following. 
Theorem 1.1. If $n \geq 2$, then

(7)

$-p^{n}{ }_{n+1} F_{n}(\lambda) \equiv\left(-\phi_{p}(-1)\right)^{n+1}\left[p^{2} X(p, \lambda, n)+p Y(p, \lambda, n)+Z(p, \lambda, n)\right] \quad\left(\bmod p^{3}\right)$

and if $n=1$, then

$$
-p_{2} F_{1}(\lambda) \equiv p^{2}[X(p, \lambda, 1)+D(p, \lambda)]+p Y(p, \lambda, 1)+Z(p, \lambda, 1) \quad\left(\bmod p^{3}\right) .
$$

We note that Theorem 1.1 generalizes both Theorem 1 in [1, where the case $n=2$ was handled modulo $p^{2}$, and Theorem 2.4 in 25. As an application of Theorem 1.1, we prove a supercongruence for the Legendre symbol $\left(\frac{-1}{p}\right)$. This result generalizes Theorem 1 in 27.

Corollary 1.2. Let $p$ be an odd prime. Then

$$
\sum_{n=0}^{\frac{p-1}{2}}\left(\begin{array}{c}
2 n \\
n
\end{array}\right)^{2} 16^{-n}+\frac{3}{8} p(-1)^{\frac{p-1}{2}} \sum_{i=1}^{\frac{p-1}{2}}\left(\begin{array}{c}
2 i \\
i
\end{array}\right) \frac{1}{i} \equiv\left(\frac{-1}{p}\right) \quad\left(\bmod p^{3}\right) .
$$

The method of proof for Theorem 1.1 has its origin in 3. Namely, the idea is to first observe that since the functions ${ }_{n+1} F_{n}(\lambda)$ are defined in terms of Jacobi sums, then one can express them as Gauss sums. One then applies the Gross-Koblitz formula [18] to express the Gauss sums in terms of $p$-adic Gamma functions. Using combinatorial properties of the $p$-adic Gamma function, Theorem 1.1 then follows. For an introduction to these methods, see 32. This general framework has been the basis for several recent results on supercongruences (see [1, 21, 25], 27], 28, 29]). Theorem 1.1 has recently been used to settle a conjecture of van Hamme (see [26]). Finally, the congruence in (9) appears to hold modulo $p^{4}$. This has been numerically confirmed for all primes less than 5000 .

The paper is organized as follows. In Section 2, we recall properties of the $p$-adic Gamma function. In Section 3, we prove Theorem 1.1. In Section 4, we prove Corollary 1.2 using Theorem 1.1 and two non-trivial Harmonic sum identities discovered using the computer summation program Sigma 39. A description of the non-trivial methods involved using the Sigma package is included in Section 5. We should also mention that similar harmonic number identities were discovered and proven in [33. These types of identities played an important role in the proof of Beukers' supercongruence for Apéry numbers (see [2] or Theorem 7 in [3]).

\section{Preliminaries}

We first recall the definition of the $p$-adic Gamma function and list some of its main properties. For more details, see 22, 30, or [37. Let $|\cdot|$ denote the $p$-adic absolute value on $\mathbb{Q}_{p}$. For $n \in \mathbb{N}$, we define

$$
\Gamma_{p}(n):=(-1)^{n} \prod_{\substack{j<n \\(j, p)=1}} j .
$$

One can extend this function to all $x \in \mathbb{Z}_{p}$ by setting

$$
\Gamma_{p}(x):=\lim _{n \rightarrow x} \Gamma_{p}(n) .
$$

The following proposition provides some of the main properties of $\Gamma_{p}$.

Proposition 2.1. Let $n \in \mathbb{N}$ and $x \in \mathbb{Z}_{p}$. Then 
(1) $\Gamma_{p}(0)=1$.

(2) $\frac{\Gamma_{p}(x+1)}{\Gamma_{p}(x)}= \begin{cases}-x & \text { if }|x|=1 \\ -1 & \text { if }|x|<1 .\end{cases}$

(3) If $0 \leq n \leq p-1$, then $n$ ! $=(-1)^{n+1} \Gamma_{p}(n+1)$.

(4) $\left|\Gamma_{p}(x)\right|=1$.

(5) Let $x_{0} \in[1,2, \ldots, p]$ be the constant term in the $p$-adic expansion of $x$. Then

$$
\Gamma_{p}(x) \Gamma_{p}(1-x)=(-1)^{x_{0}} .
$$

(6) If $x \equiv y\left(\bmod p^{n}\right)$, then $\Gamma_{p}(x) \equiv \Gamma_{p}(y)\left(\bmod p^{n}\right)$.

For $x \in \mathbb{Z}_{p}$, we define

$$
G_{1}(x):=\frac{\Gamma_{p}^{\prime}(x)}{\Gamma_{p}(x)}
$$

and

$$
G_{2}(x):=\frac{\Gamma_{p}^{\prime \prime}(x)}{\Gamma_{p}(x)} .
$$

One can check that $G_{1}(x)$ and $G_{2}(x)$ are defined for all $x \in \mathbb{Z}_{p}$ using the fact that $\Gamma_{p}(x)$ is locally analytic and $\left|\Gamma_{p}(x)\right|=1$. We now mention some congruence properties of the $p$-adic Gamma function. For a proof of this result, see [8] or [21.

Proposition 2.2. Let $p \geq 7$ be prime, $x \in \mathbb{Z}_{p}$, and $z \in p \mathbb{Z}_{p}$. Then

(1) $G_{1}(x), G_{2}(x) \in \mathbb{Z}_{p}$.

(2) We have

$$
\Gamma_{p}(x+z) \equiv \Gamma_{p}(x)\left(1+z G_{1}(x)+\frac{z^{2}}{2} G_{2}(x)\right)\left(\bmod p^{3}\right) .
$$

(3) $\Gamma_{p}^{\prime}(x+z) \equiv \Gamma_{p}^{\prime}(x)+z \Gamma_{p}^{\prime \prime}(x)\left(\bmod p^{2}\right)$.

We also need the following combinatorial congruence which relates $\Gamma_{p}$ to certain binomial coefficients.

Lemma 2.3. If $p$ is an odd prime and $1 \leq j \leq \frac{p-1}{2}$, then

$$
-\phi_{p}(-1)(-1)^{j}\left(\begin{array}{c}
\frac{p-1}{2}+j \\
j
\end{array}\right)\left(\begin{array}{c}
\frac{p-1}{2} \\
j
\end{array}\right) \equiv \frac{\Gamma_{p}\left(\frac{1}{2}+j\right)^{2}}{\Gamma_{p}(1+j)^{2}} \quad\left(\bmod p^{2}\right) .
$$

Proof. By Proposition 2.1 (3) and (5), we have

$$
\begin{aligned}
-\phi_{p}(-1)(-1)^{j}\left(\begin{array}{c}
\frac{p-1}{2}+j \\
j
\end{array}\right)\left(\begin{array}{c}
\frac{p-1}{2} \\
j
\end{array}\right) & =-\phi_{p}(-1)(-1)^{j} \frac{\left(\frac{p-1}{2}+j\right) !}{j !^{2}\left(\frac{p-1}{2}-j\right) !} \\
& =\frac{\Gamma_{p}\left(\frac{1}{2}+j+\frac{p}{2}\right) \Gamma_{p}\left(\frac{1}{2}+j-\frac{p}{2}\right)}{\Gamma_{p}(1+j)^{2}} .
\end{aligned}
$$

Now, using Proposition 2.2 (2), we have

$$
\begin{aligned}
& \Gamma_{p}\left(\frac{1}{2}+j+\frac{p}{2}\right) \Gamma_{p}\left(\frac{1}{2}+j-\frac{p}{2}\right) \\
& \equiv\left\{\Gamma_{p}\left(\frac{1}{2}+j\right)+\frac{p}{2} \Gamma_{p}^{\prime}\left(\frac{1}{2}+j\right)\right\}\left\{\Gamma_{p}\left(\frac{1}{2}+j\right)-\frac{p}{2} \Gamma_{p}^{\prime}\left(\frac{1}{2}+j\right)\right\} \quad\left(\bmod p^{2}\right) \\
& \equiv \Gamma_{p}\left(\frac{1}{2}+j\right)^{2} \quad\left(\bmod p^{2}\right)
\end{aligned}
$$

and the result follows. 
Finally, we need to define

$$
A(j):=G_{1}\left(\frac{1}{2}+j\right)-G_{1}(1+j)
$$

and for a positive integer $n$,

$$
\begin{aligned}
B(n, j):= & \frac{n+1}{2}\left(G_{2}\left(\frac{1}{2}+j\right)-G_{2}(1+j)\right)+\frac{(n+1) n}{2} G_{1}\left(\frac{1}{2}+j\right)^{2} \\
& +\frac{(n+1)(n+2)}{2} G_{1}(1+j)^{2}-(n+1)^{2} G_{1}\left(\frac{1}{2}+j\right) G_{1}(1+j) .
\end{aligned}
$$

We require the following lemma which relates $A(j)$ and $B(n, j)$ to generalized Harmonic sums. The proof is similar to Lemma 4.1 in 21] and thus is omitted.

Lemma 2.4. Let $p$ be an odd prime and $0 \leq j \leq \frac{p-1}{2}$. Then

$$
A(j) \equiv H_{\frac{p-1}{2}+j}^{(1)}-H_{j}^{(1)}+2 p \sum_{r=0}^{j-1} \frac{1}{(2 r+1)^{2}} \quad\left(\bmod p^{2}\right)
$$

and

$$
B(n, j) \equiv \frac{(n+1)^{2}}{2}\left(H_{\frac{p-1}{2}+j}^{(1)}-H_{j}^{(1)}\right)^{2}-\left(\frac{n+1}{2}\right)\left(H_{\frac{p-1}{2}+j}^{(2)}-H_{j}^{(2)}\right) \quad(\bmod p) .
$$

\section{Proof of Theorem 1.1}

We are now in a position to prove Theorem 1.1.

Proof. Let $n \geq 3$ be odd. From (1) and (2), we know that

$$
-p_{n+1}^{n} F_{n}(\lambda)=\frac{1}{1-p} \sum_{\chi} J(\phi, \chi)^{n+1} \bar{\chi}(\lambda)
$$

where $\bar{\chi}$ is the complex conjugate of $\chi$. After expressing the Jacobi sum $J(\phi, \chi)^{n+1}$ in terms of Gauss sums, we then apply the Gross-Koblitz formula [18 to get (see also [3] or [25])

(16)

$$
\begin{aligned}
-p_{n+1}^{n} F_{n}(\lambda) & =\frac{1}{1-p}\left\{\phi_{p}(\lambda)+\left(-\phi_{p}(-1)\right)^{\frac{n+1}{2}}\left(\sum_{j=0}^{\frac{p-3}{2}} \frac{\Gamma_{p}\left(\frac{j}{p-1}\right)^{n+1}}{\Gamma_{p}\left(\frac{1}{2}+\frac{j}{p-1}\right)^{n+1}} \omega^{j}(\lambda)\right.\right. \\
& \left.\left.+p^{n+1} \sum_{j=\frac{p+1}{2}}^{p-2} \frac{\Gamma_{p}\left(\frac{j}{p-1}\right)^{n+1}}{\Gamma_{p}\left(\frac{j}{p-1}-\frac{1}{2}\right)^{n+1}} \omega^{j}(\lambda)\right)\right\} .
\end{aligned}
$$

Here $\omega$ is the Teichmüller character which satisfies $\omega(\lambda) \equiv \lambda^{p^{s-1}}\left(\bmod p^{s}\right)$ and thus

$$
\omega^{j}(\lambda) \equiv \lambda^{j p^{s-1}} \quad\left(\bmod p^{s}\right)
$$

for $s \geq 1$. As $n \geq 3$ is odd, the second sum in (16) vanishes modulo $p^{3}$. As $\frac{1}{1-p} \equiv 1+p+p^{2}\left(\bmod p^{3}\right)$ and thus $\frac{j}{p-1} \equiv-j-j p-j p^{2}\left(\bmod p^{3}\right)$, we apply parts 
(5) and (6) of Proposition 2.1 and reindex the summation to obtain

$$
\begin{aligned}
-p_{n+1}^{n} F_{n}(\lambda) \equiv\left(1+p+p^{2}\right) & \left\{\phi_{p}(\lambda)+\left(-\phi_{p}(-1)\right)^{\frac{n+1}{2}}\right. \\
& \left.\cdot \sum_{j=1}^{\frac{p-1}{2}} \frac{\Gamma_{p}\left(\frac{1}{2}+j+j p+j p^{2}\right)^{n+1}}{\Gamma_{p}\left(1+j+j p+j p^{2}\right)^{n+1}} \omega^{\frac{p-1}{2}-j}(\lambda)\right\} \quad\left(\bmod p^{3}\right) .
\end{aligned}
$$

By Proposition 2.2 (2), we see that

$$
\begin{gathered}
\Gamma_{p}\left(x_{0}+j+j p+j p^{2}\right)^{n+1} \equiv \Gamma_{p}\left(x_{0}+j\right)^{n+1}\left[1+(n+1)\left(j p+j p^{2}\right) G_{1}\left(x_{0}+j\right)\right. \\
\left.+\frac{n+1}{2}\left(j p+j p^{2}\right)^{2}\left(G_{2}\left(x_{0}+j\right)+n G_{1}\left(x_{0}+j\right)^{2}\right)\right] \quad\left(\bmod p^{3}\right)
\end{gathered}
$$

for $x_{0} \in \mathbb{Z}_{p}$. We expand the numerator and denominator of (17) with $x_{0}=\frac{1}{2}$ and $x_{0}=1$ respectively. After multiplying the numerator and denominator by

$1-(n+1) j p G_{1}(1+j)-\frac{n+1}{2} j^{2} p^{2}\left(G_{2}(1+j)-(n+2) G_{1}(1+j)^{2}\right)-(n+1) j p^{2} G_{1}(1+j)$, we get

$$
\begin{aligned}
& -p_{n+1}^{n} F_{n}(\lambda) \\
& \equiv\left(1+p+p^{2}\right)\left\{\phi_{p}(\lambda)+\left(-\phi_{p}(-1)\right)^{\frac{n+1}{2}} \sum_{j=1}^{\frac{p-1}{2}} \frac{\Gamma_{p}\left(\frac{1}{2}+j\right)^{n+1}}{\Gamma_{p}(1+j)^{n+1}}(1+(n+1) j p A(j)\right. \\
& \left.\left.+(n+1) j p^{2} A(j)+j^{2} p^{2} B(n, j)\right) \omega^{\frac{p-1}{2}-j}(\lambda)\right\}\left(\bmod p^{3}\right)
\end{aligned}
$$

where $A(j)$ and $B(n, j)$ are defined by (12) and (13). We now need to consider the sums

$$
\begin{gathered}
\phi_{p}(\lambda)+\left(-\phi_{p}(-1)\right)^{\frac{n+1}{2}} \\
\cdot \sum_{j=1}^{\frac{p-1}{2}} \frac{\Gamma_{p}\left(\frac{1}{2}+j\right)^{n+1}}{\Gamma_{p}(1+j)^{n+1}}\left(1+2(n+1) j A(j)+j^{2} B(n, j)\right) \omega^{\frac{p-1}{2}-j}(\lambda), \\
\phi_{p}(\lambda)+\left(-\phi_{p}(-1)\right)^{\frac{n+1}{2}} \sum_{j=1}^{\frac{p-1}{2}} \frac{\Gamma_{p}\left(\frac{1}{2}+j\right)^{n+1}}{\Gamma_{p}(1+j)^{n+1}}(1+(n+1) j A(j)) \omega^{\frac{p-1}{2}-j}(\lambda),
\end{gathered}
$$

and

$$
\phi_{p}(\lambda)+\left(-\phi_{p}(-1)\right)^{\frac{n+1}{2}} \sum_{j=1}^{\frac{p-1}{2}} \frac{\Gamma_{p}\left(\frac{1}{2}+j\right)^{n+1}}{\Gamma_{p}(1+j)^{n+1}} \omega^{\frac{p-1}{2}-j}(\lambda)
$$

which are the coefficients of $p^{2}, p$, and 1 respectively in (18). Observe that as we want to determine ${ }_{n+1} F_{n}(\lambda) \bmod p^{3}$, it suffices to compute (19) $\bmod p$, (20) $\bmod$ $p^{2}$, and (21) $\bmod p^{3}$. Also note that

$$
\omega^{\frac{p-1}{2}-j}(\lambda)=\omega^{\frac{p-1}{2}}(\lambda) \omega^{-j}(\lambda)=\phi_{p}(\lambda) \omega^{-j}(\lambda)
$$


as $\omega$ is of order $p-1$. By Lemma 4.4 in [21, we see that

$$
\left(\frac{n+1}{2}\right) j\left(H_{\frac{p-1}{2}+j}^{(1)}-H_{\frac{p-1}{2}-j}^{(1)}\right) \equiv-2(n+1) j p \sum_{r=0}^{j-1} \frac{1}{(2 r+1)^{2}} \quad\left(\bmod p^{2}\right) .
$$

By Lemma 2.3, we have

$$
\left[-\phi_{p}(-1)(-1)^{j}\left(\begin{array}{c}
\frac{p-1}{2}+j \\
j
\end{array}\right)\left(\begin{array}{c}
\frac{p-1}{2} \\
j
\end{array}\right)\right]^{\frac{n+1}{2}} \equiv \frac{\Gamma_{p}\left(\frac{1}{2}+j\right)^{n+1}}{\Gamma_{p}(1+j)^{n+1}} \quad\left(\bmod p^{2}\right)
$$

and so after combining Lemma 2.4, (22), (23), (24) and accounting for $j=0$, then (19) is congruent modulo $p$ to (3), and (20) is congruent modulo $p^{2}$ to (4). Here we have used the fact that $\Gamma_{p}(1)^{2}=1$ and $\Gamma_{p}\left(\frac{1}{2}\right)^{2}=-\phi_{p}(-1)$ and thus for $n \geq 3$ odd

$$
\frac{\Gamma_{p}\left(\frac{1}{2}\right)^{n+1}}{\Gamma_{p}(1)^{n+1}}=\left(-\phi_{p}(-1)\right)^{\frac{n+1}{2}} .
$$

By Proposition 2.5 in [27, we have

$$
\frac{\Gamma_{p}\left(\frac{1}{2}+j\right)^{2}}{\Gamma_{p}(1+j)^{2}}=-\phi_{p}(-1)\left(\begin{array}{c}
2 j \\
j
\end{array}\right)^{2} 16^{-j}
$$

and thus using (22), we have that (21) and (5) are congruent modulo $p^{3}$, namely

$$
\begin{aligned}
\phi_{p}(\lambda)+\left(-\phi_{p}(-1)\right)^{\frac{n+1}{2}} & \sum_{j=1}^{\frac{p-1}{2}} \frac{\Gamma_{p}\left(\frac{1}{2}+j\right)^{n+1}}{\Gamma_{p}(1+j)^{n+1}} \omega^{\frac{p-1}{2}-j}(\lambda) \\
& \equiv \phi_{p}(\lambda) \sum_{j=0}^{\frac{p-1}{2}}\left(\begin{array}{c}
2 j \\
j
\end{array}\right)^{n+1} 16^{-j\left(\frac{n+1}{2}\right)} \lambda^{-j p^{2}} \quad\left(\bmod p^{3}\right) .
\end{aligned}
$$

This proves the result for $n \geq 3$ odd. A similar argument applies to the case $n \geq 2$ is even. We now turn to the case $n=1$. By (16), we need only consider the last sum

$$
-\phi_{p}(-1) p^{2} \sum_{j=\frac{p+1}{2}}^{p-2} \frac{\Gamma_{p}\left(\frac{j}{p-1}\right)^{2}}{\Gamma_{p}\left(\frac{j}{p-1}-\frac{1}{2}\right)^{2}} \omega^{j}(\lambda) .
$$

By (5) and (6) of Proposition 2.1 and after reindexing the exponent of $\omega(\lambda)$, (26) is equivalent modulo $p^{3}$ to

$$
-\phi_{p}(-1) p^{2} \sum_{j=\frac{p+1}{2}}^{p-2} \frac{\Gamma_{p}\left(\frac{1}{2}+j\right)^{2}}{\Gamma_{p}(1+j)^{2}}\left(\frac{1}{2}+j\right)^{2} \omega^{\frac{3 p-3}{2}-j}(\lambda) .
$$


By repeated use of Proposition 2.1 (2) we have for $\frac{p+1}{2} \leq j \leq p-2$ that

$$
\begin{aligned}
& \Gamma_{p}\left(\frac{1}{2}+\frac{p+1}{2}\right)^{2} \equiv 1 \quad(\bmod p) \\
& \Gamma_{p}\left(\frac{1}{2}+\frac{p+1}{2}+1\right)^{2} \equiv\left(\frac{p+2}{2}\right)^{2}(\bmod p) \\
& \Gamma_{p}\left(\frac{1}{2}+\frac{p+1}{2}+2\right)^{2} \equiv\left(\frac{p+4}{2}\right)^{2}\left(\frac{p+2}{2}\right)^{2} \quad(\bmod p) \\
& \vdots \\
& \Gamma_{p}\left(\frac{1}{2}+p-2\right)^{2} \equiv\left(\frac{2 p-5}{2}\right)^{2}\left(\frac{2 p-7}{2}\right)^{2} \cdots\left(\frac{p+2}{2}\right)^{2} \quad(\bmod p) .
\end{aligned}
$$

By Proposition 2.1 $(3), \Gamma_{p}(1+j)^{2}=(j !)^{2}$. Also using the fact that $\lambda^{p-1} \equiv 1$ $(\bmod p)$, we have

$$
\begin{aligned}
\omega^{\frac{3 p-3}{2}-j}(\lambda) & \equiv \lambda^{\frac{3 p-3}{2}-j} \quad(\bmod p) \\
& \equiv \lambda^{\frac{p-1}{2}+p-1-j} \quad(\bmod p) \\
& \equiv \lambda^{\frac{p-1}{2}-j} \quad(\bmod p)
\end{aligned}
$$

for $\frac{p+1}{2} \leq j \leq p-2$ and thus

$$
\begin{aligned}
& \sum_{j=\frac{p+1}{2}}^{p-2} \frac{\Gamma_{p}\left(\frac{1}{2}+j\right)^{2}}{\Gamma_{p}(1+j)^{2}}\left(\frac{1}{2}+j\right)^{2} \omega^{\frac{3 p-3}{2}-j}(\lambda) \\
\equiv & \frac{1}{\left(\frac{p+1}{2}\right) !^{2}}\left(\frac{p}{2}+1\right)^{2} \omega^{p-2}(\lambda)+\cdots \\
& +\frac{\left(\frac{p+2}{2}\right)^{2} \cdots\left(\frac{2 p-5}{2}\right)^{2}}{(p-2) !^{2}}\left(\frac{1}{2}+p-2\right)^{2} \omega^{\frac{p+1}{2}}(\lambda) \quad(\bmod p) \\
\equiv & \frac{1}{-\phi_{p}(-1)\left(\frac{1}{2}\right)}(1)^{2} \lambda^{-1}+\cdots \\
& +\frac{(1)^{2}(2)^{2} \cdots\left(\frac{p-5}{2}\right)^{2}}{-\phi_{p}(-1)(p-2)^{2}(p-3)^{2} \cdots\left(\frac{p+1}{2}\right)^{2}}\left(\frac{p-3}{2}\right)^{2} \lambda^{-1-\frac{p-5}{2}} \quad(\bmod p) \\
\equiv & -\phi_{p}(-1) D(p, \lambda) \quad(\bmod p) .
\end{aligned}
$$

This proves the result for $n=1$.

\section{Proof of Corollary 1.2}

Theorem 1.1 can be used to obtain modulo $p^{3}$ supercongruences in various settings. For example, Apéry numbers [3], traces of Frobenius endomorphisms on elliptic curves [24, 31], and colored partition functions [31] all occur as special values of ${ }_{n+1} F_{n}(\lambda)$ for certain $n$ and $\lambda$. We do not mention these results here, choosing instead to illustrate with one example. We now prove Corollary 1.2 . 
Proof. If $p$ is an odd prime, then by Section 3 in [16,

$$
p \cdot{ }_{2} F_{1}(1)=-\left(\frac{-1}{p}\right)
$$

and so by (8),

$$
\left(\frac{-1}{p}\right) \equiv p^{2}[X(p, 1,1)+D(p, 1)]+p Y(p, 1,1)+Z(p, 1,1) \quad\left(\bmod p^{3}\right) .
$$

We now claim that

$$
X(p, 1,1)+D(p, 1)+1 \equiv 0 \quad(\bmod p) .
$$

In order to verify (27), we first study $X(p, 1,1)$. By (3), we have

$$
\begin{array}{r}
X(p, 1,1)=\sum_{j=0}^{\frac{p-1}{2}}\left(\begin{array}{c}
\frac{p-1}{2}+j \\
j
\end{array}\right)\left(\begin{array}{c}
\frac{p-1}{2} \\
j
\end{array}\right)(-1)^{j}\left(1+4 j\left(H_{\frac{p-1}{2}+j}^{(1)}-H_{j}^{(1)}\right)\right. \\
\left.+j^{2}\left(2\left(H_{\frac{p-1}{2}+j}^{(1)}-H_{j}^{(1)}\right)^{2}-\left(H_{\frac{p-1}{2}+j}^{(2)}-H_{j}^{(2)}\right)\right)\right) .
\end{array}
$$

The identity

$$
\sum_{k=0}^{n}(-1)^{k}\left(\begin{array}{c}
n+k \\
k
\end{array}\right)\left(\begin{array}{l}
n \\
k
\end{array}\right)\left(1+2 k\left(H_{n+k}-H_{k}\right)\right)=(-1)^{n}(2 n+1)
$$

was discovered using Sigma (see Lemma 2.2 in [27]). In particular, we find

$$
\sum_{j=0}^{\frac{p-1}{2}}\left(\begin{array}{c}
\frac{p-1}{2}+j \\
j
\end{array}\right)\left(\begin{array}{c}
\frac{p-1}{2} \\
j
\end{array}\right)(-1)^{j}\left(1+2 j\left(H_{\frac{p-1}{2}+j}^{(1)}-H_{j}^{(1)}\right)\right) \equiv 0 \quad(\bmod p),
$$

and thus

$$
\begin{aligned}
X(p, 1,1) \equiv & -1-\sum_{j=1}^{\frac{p-1}{2}}\left(\begin{array}{c}
\frac{p-1}{2}+j \\
j
\end{array}\right)\left(\begin{array}{c}
\frac{p-1}{2} \\
j
\end{array}\right)(-1)^{j} \\
& +\sum_{j=1}^{\frac{p-1}{2}}\left(\begin{array}{c}
\frac{p-1}{2}+j \\
j
\end{array}\right)\left(\begin{array}{c}
\frac{p-1}{2} \\
j
\end{array}\right)(-1)^{j}\left(j ^ { 2 } \left(2\left(H_{\frac{p-1}{2}+j}^{(1)}-H_{j}^{(1)}\right)^{2}\right.\right. \\
& \left.\left.-\left(H_{\frac{p-1}{2}+j}^{(2)}-H_{j}^{(2)}\right)\right)\right) \quad(\bmod p) .
\end{aligned}
$$

By Proposition 2.2 and Lemma 2.3, we also have

$$
D(p, 1) \equiv \sum_{j=1}^{\frac{p-3}{2}}\left(\begin{array}{c}
\frac{p-1}{2}+j \\
j
\end{array}\right)^{-1}\left(\begin{array}{c}
\frac{p-1}{2} \\
j
\end{array}\right)^{-1}(-1)^{j} \quad(\bmod p) .
$$


For positive integers $n$, the relation

$$
\begin{aligned}
& \sum_{j=1}^{n}\left(\begin{array}{c}
n+j \\
j
\end{array}\right)\left(\begin{array}{c}
n \\
j
\end{array}\right)(-1)^{j}\left(j^{2}\left(2\left(H_{n+j}^{(1)}-H_{j}^{(1)}\right)^{2}-\left(H_{n+j}^{(2)}-H_{j}^{(2)}\right)\right)\right) \\
& +\sum_{j=1}^{n}\left(\begin{array}{c}
n+j \\
j
\end{array}\right)^{-1}\left(\begin{array}{c}
n \\
j
\end{array}\right)^{-1}(-1)^{j}=n(-1+2 n)(-1)^{n}
\end{aligned}
$$

was found using Sigma. Equation (27) now follows by taking $n=\frac{p-1}{2}$ in (31), in the identity

$$
\sum_{j=1}^{n}\left(\begin{array}{c}
n+j \\
j
\end{array}\right)\left(\begin{array}{l}
n \\
j
\end{array}\right)(-1)^{j}=-1+(-1)^{n},
$$

and reducing modulo $p$. We now consider $Y(p, 1,1)$. By (4), we have

$$
\begin{aligned}
Y(p, 1,1)=\sum_{j=0}^{\frac{p-1}{2}}\left(\begin{array}{c}
\frac{p-1}{2}+j \\
j
\end{array}\right)\left(\begin{array}{c}
\frac{p-1}{2} \\
j
\end{array}\right)(-1)^{j}\left(1+j\left(H_{\frac{p-1}{2}+j}^{(1)}-H_{j}^{(1)}\right)\right. \\
\left.+j\left(H_{\frac{p-1}{2}-j}^{(1)}-H_{j}^{(1)}\right)\right) .
\end{aligned}
$$

For positive integers $n$, the relation

$$
\begin{aligned}
& \sum_{j=0}^{n}\left(\begin{array}{c}
n+j \\
j
\end{array}\right)\left(\begin{array}{c}
n \\
j
\end{array}\right)(-1)^{j}\left(1+j\left(H_{n+j}^{(1)}+H_{n-j}^{(1)}-2 H_{j}^{(1)}\right)\right) \\
& =(1+2 n)\left(\begin{array}{c}
2 n \\
n
\end{array}\right)(-1)^{n}-\frac{3}{2} n(1+n)(-1)^{n} \sum_{i=1}^{n} \frac{\left(\begin{array}{c}
2 i \\
i
\end{array}\right)}{i}
\end{aligned}
$$

was discovered using Sigma. Taking $n=\frac{p-1}{2}$ in (33) and reducing $\bmod p^{2}$, we have

$$
Y(p, 1,1) \equiv p+\frac{3}{8}(-1)^{\frac{p-1}{2}} \sum_{i=1}^{\frac{p-1}{2}}\left(\begin{array}{c}
2 i \\
i
\end{array}\right) \frac{1}{i} \quad\left(\bmod p^{2}\right) .
$$

Equation (9) then follows from (5), (27), and (34).

\section{Finding and PRoving identities (31) And (33) with Sigma}

An efficient algorithm to find and prove identities involving nested definite and indefinite sum expressions, such as (31) and (33), has only recently been developed and implemented. Inspired by hypergeometric summation [34, in particular, Zeilberger's creative telescoping method [42, and Karr's indefinite summation algorithm [19, 20] (extended to definite summations), the second author developed and implemented an algorithm using Mathematica to handle various summations. The resulting package is called Sigma. For a more detailed description of the algorithms incorporated into Sigma, please see [39. Applications of this computer algebra package include proving identities that arise in the enumeration of rhombus tilings of a symmetric hexagon [12, 38, in the verification of Stembridge's totally symmetric plane partitions theorem [41, 6], and in certain Padé approximations 9 . In this section, we illustrate how the package can be used to discover and prove identities (31) and (33). For simplicity, we write $H_{k}$ for $H_{k}^{(1)}$. 
5.1. Identity (33). With Sigma we produce the following harmonic sum identities:

$$
\begin{aligned}
\sum_{k=0}^{n}(-1)^{k}\left(\begin{array}{c}
n+k \\
k
\end{array}\right)\left(\begin{array}{l}
n \\
k
\end{array}\right) k H_{k} & =(-1)^{n} n(n+1)\left(2 H_{n}-1\right), \\
\sum_{k=0}^{n}(-1)^{k}\left(\begin{array}{c}
n+k \\
k
\end{array}\right)\left(\begin{array}{l}
n \\
k
\end{array}\right) k H_{n+k} & =(-1)^{n} n(n+1) 2 H_{n}-(-1)^{n} n^{2},
\end{aligned}
$$

and

$$
\begin{aligned}
& \sum_{k=0}^{n}(-1)^{k}\left(\begin{array}{c}
n+k \\
k
\end{array}\right)\left(\begin{array}{l}
n \\
k
\end{array}\right) k H_{n-k}=-(-1)^{n}(n+1)^{2}+(-1)^{n}(2 n+1)\left(\begin{array}{c}
2 n \\
n
\end{array}\right) \\
& +2 n(n+1)(-1)^{n} H_{n}-\frac{3}{2} n(n+1)(-1)^{n} \sum_{i=1}^{n} \frac{\left(\begin{array}{c}
2 i \\
i
\end{array}\right)}{i} .
\end{aligned}
$$

Then, combining (32), (36) and (37) we arrive at identity (33).

Remark 5.1. Note that (28), (35) and (36) can be proved using hypergeometric techniques which appear in [4] and [7]. The key observation is that differentiation of the rising factorial $(x)_{k}=x(x+1) \ldots(x+k-1)$ (resp. $\left.1 /(x)_{k}\right)$ in $x$ and afterwards substituting $x=1$ produces $(1)_{k} H_{k}$ (resp. $\left.-H_{k} /(1)_{k}\right)$. With this fact, one can produce, e.g., (35) by setting up the identity

$$
\begin{aligned}
\sum_{k=0}^{n} \frac{(-n)_{k}(n+1)_{k}}{k !(x)_{k}} k & =-\frac{n(n+1)}{x}{ }_{2} F_{1}(1-n, n+2 ; x+1 ; 1) \\
& =-\frac{n(n+1)}{x} \frac{(x-n-1)_{n-1}}{(x+1)_{n-1}}
\end{aligned}
$$

with Gauss' theorem, differentiating (38) in $x$, and setting $x=1$. Obviously, the successful application of this technique relies on the fact that one knows the underlying hypergeometric identity such as (38) for the particular case (35). It would be interesting to see proofs of identity (37), in particular of identity (31), along the lines sketched above. Recently, a skillful application of partial fraction decomposition has been used in [36] to derive identity (37), but so far no proof of identity (31) has been found.

Subsequently, we illustrate the computation steps for identity (37) which can be executed in a straightforward manner. After loading the package

$\ln [1]:=<<$ Sigma.m

Sigma - A summation package by Carsten Schneider (C) RISC-Linz

into the computer algebra system Mathematica, we insert the sum in question:

$\ln [2]:=\mathbf{S}=\operatorname{SigmaSum}[\operatorname{SigmaPower}[-\mathbf{1}, \mathbf{k}] \mathbf{k S i g m a B i n o m i a l}[\mathbf{n}+\mathbf{k}, \mathbf{k}]$

SigmaBinomial $[n, k]$ SigmaHNumber $[n-k],\{k, 0, n\}]$

Out $[2]=\sum_{k=0}^{n}(-1)^{k} k\left(\begin{array}{c}n+k \\ k\end{array}\right)\left(\begin{array}{l}n \\ k\end{array}\right) H_{n-k}$

Remark 5.2. Various functions support the user, such as SigmaSum for sums, SigmaPower for powers, SigmaBinomial for binomials, or SigmaHNumber for harmonic numbers. 
Next, we compute a recurrence relation for the given sum S by inputting: $\ln [3]:=$ rec $=$ GenerateRecurrence[S]

Out $[3]=(\mathrm{n}+2)(2 \mathrm{n}+1)(\mathrm{n}+1)^{2} \operatorname{SUM}[\mathrm{n}+2]+2(\mathrm{n}+3)\left(2 \mathrm{n}^{2}+4 \mathrm{n}+1\right)(\mathrm{n}+1) \operatorname{SUM}[\mathrm{n}+1]+$

$$
(\mathrm{n}+1)(\mathrm{n}+2)(\mathrm{n}+3)(2 \mathrm{n}+3) \operatorname{SUM}[\mathrm{n}]==-(2 \mathrm{n}+1)(2 \mathrm{n}+3)(3 \mathrm{n}+1)(3 \mathrm{n}+4)(-1)^{\mathrm{n}}\left(\begin{array}{c}
2 \mathrm{n} \\
\mathrm{n}
\end{array}\right)
$$

This means that $\operatorname{SUM}[\mathrm{n}]\left(=\mathrm{S}=\sum_{k=0}^{n}(-1)^{k} k\left(\begin{array}{c}n+k \\ k\end{array}\right)\left(\begin{array}{c}n \\ k\end{array}\right) H_{n-k}\right)$ satisfies Out [3].

Proof of Out [3]. Define $f(n, k):=(-1)^{k} k\left(\begin{array}{c}n+k \\ k\end{array}\right)\left(\begin{array}{l}n \\ k\end{array}\right) H_{n-k}$. The correctness follows by the creative telescoping equation

$$
g(n, k+1)-g(n, k)=c_{0}(n) f(n, k)+c_{1}(n) f(n+1, k)+c_{2}(n) f(n+2, k)
$$

and the proof certificate

$$
\begin{gathered}
c_{0}(n)=(n+2)(n+3)(2 n+3), \\
c_{1}(n)=2(n+3)\left(2 n^{2}+4 n+1\right), c_{2}(n)=(n+1)(n+2)(2 n+1)
\end{gathered}
$$

and

$$
\begin{gathered}
g(n, k)=(k-1) k^{2}\left(2 H_{n-k}(k-n-2)(k-n-1)(n+1)\left(k(4 n+7)-2\left(2 n^{3}+10 n^{2}+17 n+10\right)\right)\right. \\
+(-k-n-1)\left(16 n^{4}+88 n^{3}+179 n^{2}+163 n+2 k^{2}\left(4 n^{2}+11 n+7\right)-k\left(24 n^{3}\right.\right. \\
\left.\left.\left.+98 n^{2}+131 n+59\right)+58\right)\right)(-1)^{k}\left(\begin{array}{c}
n+k \\
k
\end{array}\right)\left(\begin{array}{c}
n \\
k
\end{array}\right) /\left((n+1)(-k+n+1)^{2}(-k+n+2)^{2}\right)
\end{gathered}
$$

delivered by Sigma. We verify (39) as follows. Express $g(n, k+1)$ in terms of $h(n, k)=(-1)^{k}\left(\begin{array}{c}n+k \\ k\end{array}\right)\left(\begin{array}{l}n \\ k\end{array}\right)$ and $H_{n-k}$ by using the relations

$$
h(n, k+1)=-\frac{(n-k)(n+k+1)}{(k+1)^{2}} h(n, k)
$$

and

$$
H_{n-k-1}=H_{n-k}-\frac{1}{n-k} .
$$

Similarly, express $f(n+i, k)$ in terms of $h(n, k)$ and $H_{n-k}$ by using the relations

$$
h(n+1, k)=\frac{n+k+1}{n-k+1} h(n, k)
$$

and

$$
H_{n-k+1}=H_{n-k}+\frac{1}{n-k+1} .
$$

Then (39) can be checked directly. Summing (39) over $k$ from 0 to $n$ produces Out [3].

Next, we solve the recurrence relation Out 3 by typing in:

$$
\begin{aligned}
& \operatorname{In}[4]:=\operatorname{recSol}=\text { SolveRecurrence }[\operatorname{rec}[[1]], \operatorname{SUM}[\mathrm{n}]] \\
& \text { Out }[4]=\left\{\left\{0, \mathrm{n}(1+\mathrm{n})(-1)^{\mathrm{n}}\right\},\left\{0,(1+\mathrm{n})(-1)^{\mathrm{n}}\left(-1+2 \mathrm{n} \sum_{\mathrm{i}=1}^{\mathrm{n}} \frac{1}{\mathrm{i}}\right)\right\},\right. \\
& \left.\quad\left\{1,-\frac{1}{2}(-1)^{\mathrm{n}}\left(-2(1+2 \mathrm{n})\left(\begin{array}{c}
\mathrm{n} \mathrm{n} \\
\mathrm{n}
\end{array}\right)+3 \mathrm{n}(1+\mathrm{n}) \sum_{\mathrm{i}=1}^{\mathrm{n}} \frac{\left(\begin{array}{c}
2 \mathrm{i} \\
\mathrm{i}
\end{array}\right)}{\mathrm{i}}\right)\right\}\right\}
\end{aligned}
$$

The result has to be interpreted as follows. Sigma finds two linearly independent solutions $h_{1}(n)=n(1+n)(-1)^{n}$ and $h_{2}(n)=(1+n)(-1)^{n}\left(-1+2 n \sum_{i=1}^{n} \frac{1}{i}\right)$ of the 
the homogeneous version of Out [3] (indicated by the 0 in front) plus one particular solution

$$
p(n)=-\frac{1}{2}(-1)^{n}\left(-2(1+2 n)\left(\begin{array}{c}
2 n \\
n
\end{array}\right)+3 n(1+n) \sum_{i=1}^{n} \frac{\left(\begin{array}{c}
2 i \\
i
\end{array}\right)}{i}\right)
$$

of the input recurrence itself (indicated by the $1 \mathrm{in}$ front). The correctness of the result can be easily verified by using, e.g., the relation

$$
\sum_{i=1}^{n+1}\left(\begin{array}{c}
2 i \\
i
\end{array}\right) \frac{1}{i}=\sum_{i=1}^{n}\left(\begin{array}{c}
2 i \\
i
\end{array}\right) \frac{1}{i}+\frac{2(2 n+1)}{(n+1)^{2}}\left(\begin{array}{c}
2 n \\
n
\end{array}\right) .
$$

Finally, by taking all linear combinations $c_{1} h_{1}(n)+c_{2} h_{2}(n)+p(n)$ for constants $c_{1}$ and $c_{2}$, free of $n$, we obtain all solutions of Out 3 . Hence, by considering the first two initial values of $\mathrm{S}$ we can discover and prove (37):

$\ln [5]:=$ FindLinearCombination[recSol, S, 2]

Out $[5]=-(-1)^{n}(1+n)^{2}+(-1)^{n}(1+2 n)\left(\begin{array}{c}2 n \\ n\end{array}\right)+2 n(1+n)(-1)^{n} \sum_{i=1}^{n} \frac{1}{i}-\frac{3}{2} n(1+n)(-1)^{n} \sum_{i=1}^{n} \frac{\left(\begin{array}{c}2 i \\ i\end{array}\right)}{i}$

Remark 5.3. Looking at the identities (32), (36) and (35) one immediately sees that the combination (32) $+2 \cdot(36)-2 \cdot(35)$ produces (28). Since the sums can be combined so nicely, we had also hoped to find a solution for the sum

$$
S_{\lambda}(n):=\sum_{k=0}^{n}(-\lambda)^{k}\left(\begin{array}{c}
n+k \\
k
\end{array}\right)\left(\begin{array}{l}
n \\
k
\end{array}\right)\left(1+2 k\left(H_{n+k}-H_{k}\right)\right) .
$$

Sigma was able to compute the recurrence

$$
\begin{aligned}
& \quad(n+2)^{2} S_{\lambda}(n)+(2 \lambda-1)\left(4 n^{2}+18 n+21\right) S_{\lambda}(n+1) \\
& +\left(16 n^{2} \lambda^{2}+80 n \lambda^{2}+100 \lambda^{2}-16 n^{2} \lambda-80 n \lambda-100 \lambda+6 n^{2}+30 n+39\right) S_{\lambda}(n+2) \\
& +(2 \lambda-1)\left(4 n^{2}+22 n+31\right) S_{\lambda}(n+3)+(n+3)^{2} S_{\lambda}(n+4)=0,
\end{aligned}
$$

but failed to find any solution for a generic value $\lambda$. Interesting enough, choosing $\lambda=\frac{1}{2}$ the recurrences gets much simpler. In particular, this indicates that considering the sums $S_{\frac{1}{2}}(2 n)$ and $S_{\frac{1}{2}}(2 n+1)$ separately, one can compute recurrences of order 2 for each of them. Indeed, applying the mechanism from above for each of the sums gives (two different) recurrences of order two. Luckily, we can even solve the recurrences which yield

$$
S_{\frac{1}{2}}(2 n)=\frac{(-1)^{n} 2^{2 n}(n !)^{2}}{(2 n) !}
$$

and

$$
S_{\frac{1}{2}}(2 n+1)=\frac{(-1)^{n}(2 n) !}{2^{2 n}(n !)^{2}}\left((2 n+1)\left(H_{n}-H_{2 n}\right)-1\right)
$$


5.2. Identity (31). Finally, we derive the two identities

$$
\begin{gathered}
\sum_{k=1}^{n}\left(\begin{array}{c}
n+k \\
k
\end{array}\right)\left(\begin{array}{l}
n \\
k
\end{array}\right)(-1)^{k}\left(2 k^{2}\left(H_{n+k}-H_{k}\right)^{2}-k^{2}\left(H_{n+k}^{(2)}-H_{k}^{(2)}\right)\right) \\
=(1+n)^{2}\left(-2-2 n+n^{2}\right) \frac{(n !)^{2}(-1)^{n}}{2(2+2 n) !}+\frac{1}{4} n\left(-4+11 n+6 n^{2}+3 n^{3}\right)(-1)^{n} \\
\quad-\frac{1}{2}\left(-1+n+n^{2}\right)+\frac{3}{2} n^{2}(1+n)^{2}(-1)^{n} \sum_{i=1}^{n} \frac{i !^{2}}{(2+2 i) !} \\
+n^{2}(1+n)^{2}(-1)^{n} \sum_{i=1}^{n} \frac{(-1)^{i}}{i^{2}}
\end{gathered}
$$

and

(41)

$$
\begin{aligned}
\sum_{k=1}^{n} & \left(\begin{array}{c}
n+k \\
k
\end{array}\right)^{-1}\left(\begin{array}{c}
n \\
k
\end{array}\right)^{-1}(-1)^{k}=n(2 n-1)(-1)^{n} \\
& -\left((1+n)^{2}\left(-2-2 n+n^{2}\right) \frac{(n !)^{2}(-1)^{n}}{2(2+2 n) !}+\frac{1}{4} n\left(-4+11 n+6 n^{2}+3 n^{3}\right)(-1)^{n}\right. \\
& -\frac{1}{2}\left(-1+n+n^{2}\right)+\frac{3}{2} n^{2}(1+n)^{2}(-1)^{n} \sum_{i=1}^{n} \frac{i !^{2}}{(2+2 i) !} \\
& \left.+n^{2}(1+n)^{2}(-1)^{n} \sum_{i=1}^{n} \frac{(-1)^{i}}{i^{2}}\right)
\end{aligned}
$$

which immediately gives identity (31).

One option is to follow the same strategy as above: We can compute a recurrence for

$\ln [6]=\operatorname{mySum}=\sum_{\mathrm{k}=0}^{\mathrm{n}}(-1)^{\mathrm{k}}\left(\begin{array}{c}\mathrm{n}+\mathrm{k} \\ \mathrm{k}\end{array}\right)\left(\begin{array}{l}\mathbf{n} \\ \mathrm{k}\end{array}\right)\left(2 \mathrm{k}^{2}\left(\mathbf{H}_{\mathrm{n}+\mathrm{k}}-\mathbf{H}_{\mathrm{k}}\right)^{2}-\mathrm{k}^{2}\left(\mathbf{H}_{\mathrm{n}+\mathrm{k}}^{(2)}-\mathbf{H}_{\mathrm{k}}^{(2)}\right)\right)$

and we can solve the derived recurrence to find the right hand side of (40). But, since the found recurrence relation is rather large (it has order 4), and the proof certificate is even larger (it fills about one page), we follow a refined strategy presented in [33] and [40]. Namely, by running our creative telescoping algorithm with the additional option SimplifyByExt $\rightarrow$ DepthNumber we can find a recurrence of smaller order (order one!):

$$
\begin{aligned}
& \ln [7]:=\text { rec }=\text { GenerateRecurrence[mySum, SimplifyByExt } \rightarrow \text { DepthNumber }] \\
& \text { Out } 7]=2(2 n+1)(n+2)^{2} \operatorname{SUM}[n]+2(2 n+1) n^{2} \operatorname{SUM}[n+1]== \\
& 4(1+2 n)+n^{2}(n+1)(n+2)(3 n+2) \sum_{i=0}^{n} \frac{(-1)^{i}\left(\begin{array}{c}
n+i \\
i
\end{array}\right)\left(\begin{array}{c}
n \\
i
\end{array}\right)}{(n+i)^{2}}+2 n\left(4 n^{2}+3 n-4\right)(2 n+1) \sum_{i=0}^{n}(-1)^{i}\left(\begin{array}{c}
n+i+i \\
i
\end{array}\right)\left(\begin{array}{c}
n \\
i
\end{array}\right)
\end{aligned}
$$

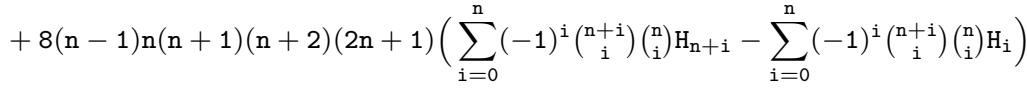

Proof of Out[7]. Define

$$
f(n, k)=(-1)^{k}\left(\begin{array}{c}
n+k \\
k
\end{array}\right)\left(\begin{array}{l}
n \\
k
\end{array}\right)\left(2 k^{2}\left(H_{n+k}-H_{k}\right)^{2}-k^{2}\left(H_{n+k}^{(2)}-H_{k}^{(2)}\right)\right) .
$$


Then the correctness of Out[7 follows by the creative telescoping equation

$$
g(n, k+1)-g(n, k)=c_{0}(n) f(n, k)+c_{1}(n) f(n+1, k)
$$

with the proof certificate $c_{0}(n)=2(n+2)^{2}(2 n+1), c_{1}(n)=2 n^{2}(2 n+1)$ and

$$
\begin{gathered}
g(n, k)=\left(4(k-1)^{2} n(n+1)^{2}(2 n+1) k^{2}\left(2 H_{k}^{2}-4 H_{n+k} H_{k}+2 H_{n+k}^{2}+H_{k}^{(2)}-H_{n+k}^{(2)}\right)\right. \\
-\left(n(n+2) k^{3}-\left(n^{3}+2 n^{2}+2 n+2\right) k^{2}-(n+1)^{2}\left(n^{2}-2\right) k+n(n+1)^{2}\left(n^{2}+n-2\right)\right) 8 n(n+1) \\
(2 n+1)\left(H_{k}-H_{n+k}\right)+\left(16 n^{5}+48 n^{4}+29 n^{3}+14 n^{2}+20 n+8\right) k^{2}+n(n+1)^{2}\left(16 n^{4}+23 n^{3}\right. \\
\left.\left.+n^{2}+12 n+8\right)-\left(32 n^{6}+101 n^{5}+98 n^{4}+55 n^{3}+54 n^{2}+36 n+8\right) k\right) \frac{(-1)^{k}\left(\begin{array}{c}
n+k \\
k
\end{array}\right)\left(\begin{array}{c}
n \\
k
\end{array}\right)}{(1-k+n) n(1+n)} \\
+n^{2}(n+1)(n+2)(3 n+2) \sum_{i=0}^{k} \frac{(-1)^{i}\left(\begin{array}{c}
n+i \\
i
\end{array}\right)\left(\begin{array}{c}
n \\
i
\end{array}\right)}{(n+i)^{2}}+2 n\left(4 n^{2}+3 n-4\right)(2 n+1) \sum_{i=0}^{k}(-1)^{i}\left(\begin{array}{c}
n+i \\
i
\end{array}\right)\left(\begin{array}{c}
n \\
i
\end{array}\right) \\
+8(n-1) n(n+1)(n+2)(2 n+1)\left(\sum_{i=0}^{k}(-1)^{i}\left(\begin{array}{c}
n+i \\
i
\end{array}\right)\left(\begin{array}{c}
n \\
i
\end{array}\right) H_{n+i}-\sum_{i=0}^{k}(-1)^{i}\left(\begin{array}{c}
n+i \\
i
\end{array}\right)\left(\begin{array}{c}
n \\
i
\end{array}\right) H_{i}\right) .
\end{gathered}
$$

Since the sums and products inside of $g(n, k)$ are all indefinite, e.g., we can apply the relation

$$
\sum_{i=0}^{k+1}(-1)^{i}\left(\begin{array}{c}
n+i \\
i
\end{array}\right)\left(\begin{array}{c}
n \\
i
\end{array}\right)=\sum_{i=0}^{k}(-1)^{i}\left(\begin{array}{c}
n+i \\
i
\end{array}\right)\left(\begin{array}{c}
n \\
i
\end{array}\right)-(-1)^{k} \frac{(k-n)(k+n+1)}{(k+1)^{2}}\left(\begin{array}{c}
n+k \\
k
\end{array}\right)\left(\begin{array}{c}
n \\
k
\end{array}\right),
$$

the verification of (42) is immediate. Summing (42) over $k$ from 0 to $n$ produces Out[7].

At first glance the recurrence Out[7 seems to be disappointing, we start with the definite sum mySum, and end up with a recurrence again involving definite sums. But, these sums are much simpler than the input sum. In particular, facilitating again Sigma, we can produce mechanically the identities

$$
\sum_{i=0}^{n}(-1)^{i} \frac{\left(\begin{array}{c}
n+i \\
i
\end{array}\right)\left(\begin{array}{c}
n \\
i
\end{array}\right)}{(n+i)^{2}}=-(-1)^{n} \frac{n !^{2}}{n^{2}(2 n) !}
$$

and

$$
\sum_{k=0}^{n}(-1)^{k}\left(\begin{array}{c}
n+k \\
k
\end{array}\right)\left(\begin{array}{l}
n \\
k
\end{array}\right) H_{k}=\sum_{k=0}^{n}(-1)^{k}\left(\begin{array}{c}
n+k \\
k
\end{array}\right)\left(\begin{array}{l}
n \\
k
\end{array}\right) H_{n+k}=(-1)^{n} 2 H_{n} .
$$

Using in addition (32), we can simplify the recurrence to

$$
\begin{aligned}
& \ln [8]:=\operatorname{rec}=\operatorname{rec} / \cdot\left\{\sum_{i=0}^{n}(-1)^{i} \frac{\left(\begin{array}{c}
n+i \\
i
\end{array}\right)\left(\begin{array}{c}
n \\
i
\end{array}\right)}{(n+i)^{2}} \rightarrow-(-1)^{n} \frac{n !^{2}}{n^{2}(2 n) !}, \sum_{i=0}^{n}(-1)^{i}\left(\begin{array}{c}
n+i \\
i
\end{array}\right)\left(\begin{array}{c}
n \\
i
\end{array}\right) \rightarrow(-1)^{n},\right. \\
& \left.\sum_{i=0}^{n}(-1)^{i}\left(\begin{array}{c}
n+i \\
i
\end{array}\right)\left(\begin{array}{c}
n \\
i
\end{array}\right) H_{i} \rightarrow \sum_{i=0}^{n}(-1)^{i}\left(\begin{array}{c}
n+i \\
i
\end{array}\right)\left(\begin{array}{c}
n \\
i
\end{array}\right) H_{n+i}\right\} \\
& \text { Out }[8]=2(2 \mathrm{n}+1) \mathrm{n}^{2} \operatorname{SUM}[\mathrm{n}+1]+2(\mathrm{n}+2)^{2}(2 \mathrm{n}+1) \operatorname{SUM}[\mathrm{n}]== \\
& 4(2 n+1)-\frac{(-1)^{n}(n+1)(n+2)(3 n+2)(n !)^{2}}{(2 n) !}+2(-1)^{n} n(2 n+1)\left(4 n^{2}+3 n-4\right) .
\end{aligned}
$$

Given this recurrence, one can directly see its solution. With some simplifications Sigma yields: 


$$
\begin{aligned}
\operatorname{In}[9]:= & \text { recSol }=\text { SolveRecurrence }[\operatorname{rec}[[1]], \text { SUM }[\mathbf{n}], \text { SimpleSumRepresentation } \rightarrow \text { True }] \\
\text { Out }[9]= & \left\{\left\{0,(-1)^{n} n^{2}(n+1)^{2},\left\{1,(1+n)^{2}\left(-2-2 n+n^{2}\right) \frac{(n !)^{2}(-1)^{n}}{2(2+2 n) !}-\frac{1}{2}\left(-1+n+n^{2}\right)\right.\right.\right. \\
& \left.\left.+\frac{1}{4} n\left(-4+11 n+6 n^{2}+3 n^{3}\right)(-1)^{n}+\frac{3}{2} n^{2}(1+n)^{2}(-1)^{n} \sum_{i=1}^{n} \frac{i !}{(2+2 i) !}+n^{2}(1+n)^{2}(-1)^{n} \sum_{i=1}^{n} \frac{(-1)^{i}}{i^{2}}\right\}\right\}
\end{aligned}
$$

Looking at the first initial values we end up at the identity (40).

Finally, we attack the sum $S(n)$ on the left hand side of (41). Namely, we derive the recurrence

$$
(n+2) S(n)+n^{2} S(n)=\frac{(-1)^{n}(n+1)^{2}(n+2)(3 n+2)(n !)^{2}}{(2 n+2) !}-2
$$

we remark that for this hypergeometric sum any implementation of Zeilberger's algorithm 42 could do the job. Using this information, we find as above, the right hand side of (41).

\section{ACKNOWLEDGMENTS}

The first author would like to thank the Institut des Hautes Études Scientifiques for their hospitality and support during the preparation of this paper. The authors also thank Ken Ono for his comments on a preliminary version of the paper, Dermot McCarthy for his careful reading, and the referee for helpful suggestions.

\section{REFERENCES}

[1] S. Ahlgren, Gaussian hypergeometric series and combinatorial congruences, Symbolic computation, number theory, special functions, physics and combinatorics (Gainesville, Fl, 1999), 1-12, Dev. Math., 4, Kluwer, Dordrecht, 2001. MR 1880076 (2003i:33025)

[2] S. Ahlgren, S. Ekhad, K. Ono, D. Zeilberger, A binomial coefficient identity associated to a conjecture of Beukers, Electron. J. Combin. 5 (1998), Research Paper 10, 1 p. MR1600106

[3] S. Ahlgren, K. Ono, A Gaussian hypergeometric series evaluation and Apéry number congruences, J. Reine Angew. Math. 518 (2000), 187-212. MR1739404 (2001c:11057)

[4] G. E. Andrews, Applications of basic hypergeometric functions, SIAM Rev. 16 (1974), 441484. MR0352557 (50:5044)

[5] G. Andrews, R. Askey, R. Roy, Special Functions, Encyclopedia of Mathematics and its Applications, 71. Cambridge University Press, Cambridge, 1999. MR 1688958 (2000g:33001)

[6] G. Andrews, P. Paule, C. Schneider, Plane partitions. VI. Stembridge's TSPP theorem, Adv. in Appl. Math. 34 (2005), no. 4, 709-739. MR2128995 (2006b:05012)

[7] G. E. Andrews and K. Uchimura, Identities in combinatorics. IV. Differentiation and harmonic numbers, Utilitas Math. 28 (1985), 265-269. MR821962 (87e:05014)

[8] S. Chowla, B. Dwork, and R. Evans, On the mod $p^{2}$ determination of $\left(\begin{array}{l}(p-1) / 2 \\ (p-1) / 4\end{array}\right)$, J. Number Th. 24 (1986), no. 2, 188-196. MR863654 (88a:11130)

[9] K. Driver, H. Prodinger, C. Schneider, J. Weideman, Padé approximations to the logarithm. II. Identities, recurrences, and symbolic computation, Ramanujan J. 11 (2006), no. 2, 139158. MR2267670 (2007i:05017)

[10] R. Evans, Identities for products of Gauss sums over finite fields, Enseign. Math. 27 (1981), 197-209. MR659148 (83i:10050)

[11] S. Frechette, K. Ono, and M. Papanikolas, Gaussian hypergeometric functions and traces of Hecke operators, Int. Math. Res. Not. 2004, no. 60, 3233-3262. MR2096220(2006a:11055)

[12] M. Fulmek, C. Krattenthaler, The number of rhombus tilings of a symmetric hexagon which contain a fixed rhombus on the symmetry axis. II, European J. Combin. 21 (2000), no. 5, 601-640. MR1771987(2002e:05039)

[13] J. Fuselier, Hypergeometric functions over finite fields and relations to modular forms and elliptic curves, Ph.D. thesis, Texas A\&M University, 2007. 
[14] I. Gel'fand, M. Graev, Hypergeometric functions over finite field, Dokl. Akad. Nauk 381 (2001), no. 6, 732-737. MR.1892519 (2003f:33020)

[15] I. Gel'fand, M. Graev, and V. Retakh, Hypergeometric functions over an arbitrary field, (Russian) Uspekhi Mat. Nauk 59 (2004), no. 5 (359), 29-100; translation in Russian Math. Surveys 59 (2004), no. 5, 831-905. MR2125927(2006e:33022)

[16] J. Greene, Character sum analogues for hypergeometric and generalized hypergeometric functions over finite fields, Ph.D. thesis, University of Minnesota, 1984.

[17] J. Greene, Hypergeometric series over finite fields, Trans. Amer. Math. Soc. 301 (1987), 77-101. MR879564 (88e:11122)

[18] B. Gross, N. Koblitz, Gauss sums and the p-adic $\Gamma$-function, Ann. of Math. (2) 109 (1979), no. 3, 569-581. MR534763 (80g:12015)

[19] M. Karr, Summation in finite terms, J. Assoc. Comput. Mach. 28 (1981), no. 2, 305-350. MR.612083(82m:12018)

[20] M. Karr, Theory of summation in finite terms, J. Symbolic Comput. 1 (1985), no. 3, 303-315. MR849038(89a:12016)

[21] T. Kilbourn, An extension of the Apéry number supercongruence, Acta Arith. 123 (2006), 335-348. MR2262248 (2007e:11049)

[22] N. Koblitz, p-adic numbers, p-adic analysis, and zeta functions, Second Edition. Graduate Texts in Mathematics 58, Springer-Verlag, New York, 1984. MR754003 (86c:11086)

[23] N. Koblitz, The number of points on certain families of hypersurfaces over finite fields, Compositio Math. 48 (1983), 3-23. MR700577 (85d:14034)

[24] M. Koike, Orthogonal matrices obtained from hypergeometric series over finite fields and elliptic curves over finite fields, Hiroshima Math. J. 25 (1995), 43-52. MR1322601 (96b:11079)

[25] P. Loh, R. Rhoades, $p$-adic and combinatorial properties of modular form coefficients, Int. J. Number Theory 2 (2006), no. 2, 305-328. MR2240232 (2007f:11047)

[26] D. McCarthy, R. Osburn, A p-adic analogue of a formula of Ramanujan, submitted.

[27] E. Mortenson, A supercongruence conjecture of Rodriguez-Villegas for a certain truncated hypergeometric function, J. Number Th. 99 (2003), no. 1, 139-147. MR.1957248(2004e:11089)

[28] E. Mortenson, Supercongruences between truncated ${ }_{2} F_{1}$ hypergeometric functions and their Gaussian analogs, Trans. Amer. Math. Soc. 355 (2003), 987-1007. MR1938742 (2003i:11119)

[29] E. Mortenson, Supercongruences for truncated ${ }_{n+1} F_{n}$ hypergeometric series with applications to certain weight three newforms, Proc. Amer. Math. Soc. 133 (2005), no. 2, 321-330. MR2093051 (2005f:11080)

[30] R. Murty, Introduction to p-adic analytic number theory, AMSCIP Studies in Advanced Mathematics, vol. 27, American Mathematical Society, Providence, RI, 2002. MR 1913413 (2003c:11151)

[31] K. Ono, Values of Gaussian hypergeometric series, Trans. Amer. Math. Soc. 350 (1998), 1205-1223. MR1407498 (98e:11141)

[32] K. Ono, The web of modularity: arithmetic of the coefficients of modular forms and q-series, Amer. Math. Soc., CBMS Regional Conf. in Math., vol. 102, 2004. MR2020489|(2005c:11053)

[33] P. Paule, C. Schneider, Computer proofs of a new family of harmonic number identities, Adv. in Appl. Math. 31 (2003), no. 2, 359-378. MR2001619 (2004f:33043)

[34] M. Petkovšek, H. S. Wilf, and D. Zeilberger, $A=B$, A. K. Peters, Wellesley, MA, 1996. MR:1379802 (97j:05001)

[35] M. Papanikolas, A formula and a congruence for Ramanujan's $\tau$-function, Proc. Amer. Math. Soc. 134 (2006), no. 2, 333-341. MR2175999 (2007d:11046)

[36] H. Prodinger, Human proofs of identities by Osburn and Schneider, preprint available at http://front.math.ucdavis.edu/0710.0464.

[37] A. Robert, A course in p-adic analysis, Graduate Texts in Mathematics 198, Springer-Verlag, New York, 2000. MR1760253 (2001g:11182)

[38] C. Schneider, The summation package Sigma: Underlying principles and a rhombus tiling application, Discrete Math. Theor. Comput. Sci., 6 (2004), no. 2, 365-386. MR2081481 (2005e:68270)

[39] C. Schneider, Symbolic Summation Assists Combinatorics, Sem. Lothar. Combin., 56:1-36, 2007. Article B56b. MR2317679

[40] C. Schneider, A refined difference field theory for symbolic summation, SFB-Report 2007-24, SFB F013, J. Kepler University Linz, 2007. 
[41] J. Stembridge, The enumeration of totally symmetric plane partitions, Adv. Math. 111 (1995), no. 2, 227-243. MR 1318529 (96b:05010)

[42] D. Zeilberger, The method of creative telescoping, J. Symbolic Comput. 11 (1991), no. 3, 195-204. MR1103727 (92c:33005)

[43] K. Yamamoto, On a conjecture of Hasse concerning multiplicative relations of Gaussian sums, J. Combin. Theory Ser. A 1 (1966), 476-489. MR0213311(35:4175)

School of Mathematical Sciences, University College Dublin, Belfield, Dublin 4, IRELAND

E-mail address: robert.osburn@ucd.ie

Current address: IHÉS, Le Bois-Marie, 35, route de Chartres, F-91440 Bures-sur-Yvette, France

E-mail address: osburn@ihes.fr

Research Institute for Symbolic Computation, J. Kepler University Linz, Altenberger Strasse 69, A-4040 Linz, Austria

E-mail address: Carsten.Schneider@risc.uni-linz.ac.at 